\title{
A pós-graduação stricto sensu em educação física no Rio de Janeiro: Uma análise genealógica foucaultiana
}

\author{
The stricto sensu graduate program in physical education in Rio de Janeiro: A foucault's \\ genelogical analysis \\ El posgrado stricto sensu en educación física en Río de Janeiro: Un análisis genealógico \\ foucaultiano
}

Recebido: 01/07/2021 | Revisado: 06/07/2021 | Aceito: 09/07/2021 | Publicado: 21/07/2021

\author{
Renata Ferreira Chrispino \\ ORCID: https://orcid.org/0000-0002-8443-8821 \\ Prefeitura Municipal do Rio de Janeiro, Brasil \\ E-mail: renatachrispino@hotmail.com \\ Camila Regina Soares da Silva \\ ORCID: https://orcid.org/0000-0002-1464-9666 \\ Prefeitura Municipal do Rio de Janeiro, Brasil \\ E-mail: camilaef@hotmail.com \\ Flavia Fernandes de Oliveira \\ ORCID: https://orcid.org/0000-0002-3615-6846 \\ Universidade do Estado do Rio de Janeiro, Brasil \\ E-mail: tabininha@gmail.com \\ Felipe da Silva Triani \\ ORCID: https://orcid.org/0000-0001-6470-8823 \\ Universidade do Estado do Rio de Janeiro, Brasil \\ E-mail: felipetriani@gmail.com \\ Silvio de Cassio Costa Telles \\ ORCID: https://orcid.org/0000-0003-2652-6118 \\ Universidade do Estado do Rio de Janeiro, Brasil \\ E-mail: silviotelles@terra.com.br \\ Rafael da Silva Mattos \\ ORCID https://orcid.org/0000-0002-1314-050X \\ Universidade do Estado do Rio de Janeiro, Brasil \\ E-mail: profmattos2010@gmail.com
}

\begin{abstract}
Resumo
A concepção biologizante da Educação Física é o conceito restrita entre a saúde e a biologia, que se perpetua por muito tempo na área para dar conta do status quo. O presente artigo tem como objetivo investigar a representatividade das subáreas da educação física dentro dos cursos de pós-graduação stricto sensu no Rio de Janeiro e discutir os efeitos desta distribuição na constituição do campo, a partir da análise genealógica foucaultiana. Para tal foi realizado um levantamento nos três programas de pós-graduação stricto-sensu do Rio de Janeiro, que buscou coletar dados do quadriênio (2017-2020), número de docentes, dissertações e teses defendidas ou em andamento nesse período. Concluise que diante da genealogia foucaultiana em que o saber-poder na discursividade da biologização da Educação Física nos permite compreender que a Educação Física se aproxima do modelo hegemônico de ciência, conquistando maior legitimidade nos campos científico e social.
\end{abstract}

Palavras-chave: Ensino; Pós-graduação; Educação física; Genealogia.

\begin{abstract}
The biologic conception of Physical Education is the restricted concept between health and biology, which is perpetuated for a long time in the area to account for the status quo. This article aims to investigate the representativeness of the physical education sub-areas within the stricto sensu graduate courses in Rio de Janeiro and to discuss the effects of this distribution on the constitution of the field, based on Foucault's genealogical analysis. For this purpose, a survey was carried out in the three stricto-sensu graduate programs in Rio de Janeiro, which sought to collect data on the fouryear period (2017-2020), number of teachers, dissertations and theses defended or in progress during this period. It is concluded that given the Foucault's genealogy in which the knowledge-power in the discursivity of the biologization of Physical Education allows us to understand that Physical Education approaches the hegemonic model of science, achieving greater legitimacy in the scientific and social fields.
\end{abstract}

Keywords: Teaching; Graduate; Physical education; Genealogy. 


\section{Resumen}

El concepto biologizante de Educación Física es el concepto restringido entre salud y biología, que se ha perpetuado durante mucho tiempo en el área para dar cuenta del statu quo. Este artículo tiene como objetivo investigar la representatividad de las subáreas de educación física dentro de los posgrados stricto sensu en Río de Janeiro y discutir los efectos de esta distribución en la constitución del campo, con base en el análisis genealógico de Foucault. Para ello, se realizó una encuesta en los tres programas de posgrado stricto-sensu en Río de Janeiro, que buscó recolectar datos del cuatrienio (2017-2020), número de profesores, disertaciones y tesis defendidas o en curso durante ese período. Se concluye que dada la genealogía foucaultiana en la que el saber-poder en la discursividad de la biologización de la Educación Física permite comprender que la Educación Física se acerca al modelo hegemónico de la ciencia, logrando una mayor legitimidad en los campos científico y social.

Palabras clave: Enseñanza; Posgraduación; Educación física; Genealogía.

\section{Introdução}

A área da Educação Física originou-se das concepções naturalistas de homem e corpo que se desenvolveram, sobretudo no século XIX. A presença de médicos como os profissionais responsáveis pela prescrição de exercícios físicos conferiu a princípio uma característica biologizante para a área. Objetivava-se um corpo livre de doenças em um contexto de eugenia e higienia. A Educação Física, no final do século XIX, "foi entendida como um elemento de extrema importância para o forjar daquele indivíduo 'forte', 'saudável', indispensável à implementação do processo de desenvolvimento do país” (Castellani Filho, 1988, p. 39). Naquela época o modelo de sociedade capitalista, se limitava em um padrão de corpo saudável e robusto, livre de doenças e produtivo, que para Paiva (2004, p.54) teriam deflagrado um processo de biologização da Educação Física, sempre operando com um conceito restrito de saúde - biológica.

A Educação Física, que no Brasil foi fortemente influenciada pela Medicina Higienista do século XIX e desde então ao longo de sua construção com as tendências higienista, militarista, pedagogicista, competitivista, esteve comprometida com a ideologia dominante, por meio da prática corporal do esporte (Bracht, 1997). O esporte desde então passa a ser o vínculo entre Educação Física e sociedade moderna, conquistando os olhares das Ciências do Esporte no interior das universidades.

Até os dias atuais a Educação Física tem apresentado dificuldade na afirmação de sua identidade enquanto ciência ou profissão reconhecida legalmente. É uma área de conhecimento que recebe influências de diversas áreas, como as ciências naturais, humanas e sociais (Manoel \& Carvalho, 2011). Segundo esses autores, desde o final dos anos de 1970, quando se iniciaram os processos de criação dos programas de pós-graduação em Educação Física muito se discute a identidade acadêmica da área.

A Educação Física tem um papel importante no desenvolvimento da aptidão física. Contudo, também tem características educacionais voltadas para a formação psicossocial do indivíduo, mesmo constituindo um ramo complexo de conhecimentos, que engloba disciplinas situadas predominantemente no campo biomédico, partilhando inclusive o seu quadro epistemológico (Luz, 2007).

Apesar de ser um componente distinto, a pós-graduação está fortemente relacionado a educação superior de graduação, por ser considerada primordialmente como um meio de formação de seus docentes, e também com as atividades de pesquisa e desenvolvimento, capacitando recursos humanos para atuar nesta área de produção de conhecimento (Kokubun, 2006; Triani, Magalhães Júnior, Novikoff, 2017).

Muitos foram os debates e modificações sofridas pelos cursos de pós-graduação ao longo dos anos (Lüdorf, 2002). De acordo com Manoel e Carvalho (2011), no final da década de 1990 os programas de pós-graduação em educação física passaram por um processo de reestruturação, fazendo emergir três subáreas, a saber: biodinâmica, sociocultural e pedagógica. A primeira faz referência ao campo em que a biologia é a base epistemológica; a segunda trata-se do paradigma no qual as ciências sociais e humanas compõem o referencial teórico de produção do conhecimento; e a terceira é a subárea que compreende os fenômenos a partir das ciências da educação. 
De acordo com o Fórum de Pesquisadores das Subáreas Sociocultural e Pedagógica (2015) há um descompasso entre as três subáreas, sendo a biodinâmica a representante de mais de 70\% do total de capital científico da área. Diante disso, o estudo também desvela que a Educação Física se expandiu enquanto campo, porém, afastou-se dos debates pedagógicos na medida em que a subárea pedagógica não se alargou nas mesmas proporções que a biodinâmica.

Dar-se aí o objetivo deste artigo que é investigar a representatividade das subáreas da educação física dentro dos cursos de pós-graduação stricto sensu no Rio de Janeiro e discutir os efeitos desta distribuição na constituição do campo, a partir da análise genealógica foucaultiana. A genealogia do poder de Foucault (2014) seria relação entre o discurso e o poder, ou seja, é a estratégia da discursividade dos saberes.

\section{Metodologia}

A metodologia trata-se de uma pesquisa qualitativa, exploratória e descritiva (Thomas, Nelson \& Silverman, 2012). Para a análise dos dados deste estudo foi realizado um levantamento nos três programas de pós-graduação stricto-sensu do Rio de Janeiro, a saber: Programa de Pós-Graduação em Educação Física da Universidade Federal do Rio de Janeiro - PPGEF-UFRJ; Programa de Pós-graduação em Ciências da Atividade Física da Universidade Salgado de Oliveira - PPGAF-UNIVERSO; e Programa de Pós-Graduação em Ciências do Exercício e do Esporte da Universidade do Estado do Rio de Janeiro - PPGCEEUERJ.

A coleta de dados foi realizada a partir das informações dos Programas de Pós-graduação em Educação Física do Rio de Janeiro disponibilizadas em seus respectivos sites. Além disso, para o levantamento do número de dissertações e teses foi realizada a busca de todos os docentes permanentes dos programas e em seguida acessado o currículo lattes de cada um deles. Cabe ressaltar que o levantamento dos dados ocorreu no quadriênio (2017-2020), sendo a coleta realizada de 2017 até o primeiro semestre de 2019, abrangendo as dissertações e teses defendidas ou em andamento nesse período.

O método genealógico foulcaultiano consiste em um instrumental de investigação voltado à compreensão da emergência de sujeitos, objetos e significações nas relações de poder-saber. Ressalta-se que método aqui se refere a uma análise e interpretação histórico-filosófica, pois Foucault não era um cientista que submetia suas reflexões a experimentos, na linguagem científica propriamente dita. Para Foucault (2008), o conhecimento se constitui a partir de relações de saber-poder e procura identificar as condições de possibilidade de certos objetos do discurso que se constituem como " a verdade", tal como fez com o conceito de loucura, com a constituição do conceito de anormal, entre outros. O discurso é produto da sua época, do poder e saber de seu tempo. Logo, é necessário um método que seja histórico, sem ser positivista ou meta-histórico. Para Foucault (1989), as práticas discursivas tomam corpo no seu conjunto de técnicas, nas instituições, nos esquemas de comportamento, nos seus tipos de transmissão e de difusão, nas formas pedagógicas que ao mesmo tempo se impõem e se mantém.

\section{Resultados e Discussão}

A organização dos dados coletados ocorreu por meio de três categorias de análise que foram eleitas, isto é, cada subárea da educação física corresponde a uma categoria de análise. Desse modo, o número de docente e as dissertações e teses foram distribuídas por subárea da Educação Física. 
Tabela 1. Quantitativo de Docentes, dissertações e teses dos programas de Pós-Graduação em Educação Física do Rio de Janeiro por subárea até o primeiro semestre de 2019.

\section{Subáreas}

\begin{tabular}{lllllllll}
\cline { 4 - 6 } Variáveis & & Total & & & Biodinâmica & \multicolumn{2}{c}{ Sociocultural } & \multicolumn{2}{c}{ Pedagógica } \\
\cline { 5 - 8 } & $\mathrm{n}$ & $\%$ & $\mathrm{n}$ & $\%$ & $\mathrm{~N}$ & $\%$ & $\mathrm{n}$ & $\%$ \\
\hline
\end{tabular}

Docentes Permanentes

\begin{tabular}{|c|c|c|c|c|c|c|c|}
\hline UERJ & 16 & 34 & 13 & 81 & $3 *$ & 19 & $3 *$ \\
\hline UFRJ & 19 & 40 & 17 & 89 & $2 *$ & 11 & $2 *$ \\
\hline UNIVERSO & 12 & 26 & 6 & 50 & $6^{*}$ & 50 & $6^{*}$ \\
\hline
\end{tabular}

Dissertações e Teses

\begin{tabular}{lcccccccc} 
UERJ & 69 & 24 & 52 & 69 & 11 & 16 & 6 & 8 \\
UFRJ & 116 & 41 & 99 & 85 & 13 & 11 & 4 & 3 \\
UNIVERSO & 101 & 35 & 62 & 61 & 32 & 32 & 7 & 7 \\
\hline
\end{tabular}

Legenda: * De acordo com as informações disponibilizadas no site dos programas, os professores que não orientam na biodinâmica, orientam nas subáreas pedagógica e sociocultural. Fonte: Autores.

Analisando a tabela acima, verifica-se que no período de 2017 até o primeiro semestre de 2019, nota-se que na UERJ, $81 \%$ dos docentes permanentes são da subárea biodinâmica, já na UFRJ e na UNIVERSO 89\% e 50\% são da subárea biodinâmica respectivamente, por outro lado 19\%,11\% e 50\% são das subáreas sociocultural e pedagógica. Dentre essas três universidades, apenas a Universo apresenta proporcionalidade entre o número de docentes da subárea biodinâmica e sociocultural, pedagógica. Dessa forma, a porcentagem de dissertações e teses apresentadas neste mesmo período, segue a proporção de docentes. Na UERJ, 69\% das dissertações e teses apresentadas são da subárea biodinâmica, 16 \% da subárea sociocultural e $8 \%$ da subárea pedagógica. Na UFRJ, $85 \%$ da biodinâmica, $11 \%$ da sociocultural e 3\% da pedagógica. Na Universo, $61 \%$ das dissertações e teses são da biodinâmica, 32\% da sociocultural e apenas 7 \% da pedagógica. Assim mesmo a Universo apresentando no quadro de docentes uma certa proporcionalidade entre as subáreas, há uma discrepância entre os números de dissertações e teses entre as subáreas. São $61 \%$ da biodinâmica, $32 \%$ da sociocultural e $7 \%$ da pedagógica.

A educação física parecer estar fazendo uma opção evidente pela subárea biodinâmica e assim deve se consolidar, retirando a área sociocultural e pedagógica de seu campo. Nesse sentido, seria mais interessante que os docentes dessas subáreas e os discentes fossem absorvidos por outros programas, mas não os de Educação Física. Se a Educação Física opta por se intervir na lógica da produtividade, não faz sentido a existência das subáreas sociocultural e pedagógica. Nesse sentido, os docentes e discentes deveriam se inserir em programas de história, sociologia, filosofia, educação, etc.

Contudo, o problema disso é a descaracterização da Educação Física enquanto prática pedagógica que tematiza a cultura corporal e que tem, dentre seus campos de atuação profissional, o ambiente escolar. Assim, a Educação Física tornar-se-ia um apêndice da Medicina e da Biologia.

A genealogia do poder de Foucault (2014) vai de encontro aos discursos sobre a suposição de dominação desta área, em que os saberes históricos são predominantes, esta oposição se dá através da luta de um discurso único e científico. Toda a produção de conhecimento desta subárea são frutos do modo de vida em que todos estão inseridos, como o efeito da biologização da Educação Física se dá pela apropriação do discurso científico de saúde através de uma técnica de poder nomeada por Foucault 
(1999) como "biopolítica". As regras dominantes na formação das condições da existência são também da coexistência, manutenção, modificação e desaparição de um determinado campo do discurso, segundo Williams (2012).

A Bipolítica é a prática de biopoderes locais, em que a população é tanto alvo como instrumento em uma relação de poder. "Os instrumentos que o governo se dará para obter esses fins [atendimento as necessidades e desejos da população] que são, de algum modo, imanentes ao campo da população, serão essencialmente a população sobre o qual ele age" (Foucault, 2014).

Esta subárea corresponde às pesquisas que tem como determinantes em suas linhas a fisiologia humana, a aprendizagem motora, a biomecânica e a saúde, que são as exigências da contemporaneidade científica. Esse discurso ideológico é o da Performance Humana, que busca cientificamente verificar os efeitos do exercício para o aumento ou a melhora do poder sobre a vida ou a gestão da vida. Este é o principal alvo da biopolítica o conjunto dos indivíduos da população e designa os mecanismos estratégicos de poder sobre a vida e sua gestão. Portanto, sobre essa análise, a biologização dos Programas de Pós-Graduação em Educação Física é uma estratégia biopolítica vitoriosa, pelo menos no Estado do Rio de Janeiro.

Em estudo recente (Triani et al. 2019), cujo objetivo foi identificar as representações sociais compartilhadas por um grupo de estudantes de educação física, foi observado que o discurso da saúde, em uma perspectiva biológica, corresponde ao núcleo central das representações sociais que os acadêmicos compartilhavam sobre a educação física. A investigação demonstrou que o grupo participante da pesquisa desconsiderada a dimensão humanística da área. Ressalta-se que os currículos dos cursos de graduação não sofrem imposições de disciplinas obrigatórias, permitindo, assim, que os próprios docentes dos cursos façam propostas de cursos de Educação Física com abordagem sociocultural ou pedagógica. Evidencia-se que o campo institucional de ensino superior opta voluntariamente pela biologização da Educação Física, não podendo alegar interferência do Estado ou de governos.

Ainda nessa perspectiva, Triani e Telles (2019) estudaram a produção científica da educação física a partir dos periódicos especializados da área. Na ocasião, foram analisadas 2083 revistas que publicam artigos da educação física. Essa análise demonstrou que do total de periódicos de veiculação de conhecimento científico na área, $70 \%$ são específicos da biodinâmica. Esse cenário, reforça a ideia de que até no meio científico a representatividade da biodinâmica vem sendo superior, implicam em uma desproporcionalidade no campo e enfraquecendo a educação física como área que se caracteriza por sua diversidade epistemológica. Quem avalia quais artigos serão publicados são os próprios docentes da área, sobretudo, aqueles que atuam em Programas de Pós-Graduação. Logo, percebe-se que os próprios docentes supervalorizam os estudos biomédicos e biodinâmicos. Aqui não se pode recorrer a pressão de agências de fomento ou de governos.

Segundo Telles, Lüdorf e Pereira (2017), dentro da área sociocultural, que aborda temas como práticas corporais, esportes e atividades físicas nas perspectivas da sociologia, da antropologia, da filosofia e da história, as concepções de ciência, pesquisa e métodos, se diferem do modo de pensar oriundo das ciências naturais, amparadas pelos pressupostos da ciência tradicional, não melhores ou piores, mas diferentes, que impactam nas formas de avaliação. Além disso muitas das vezes as pesquisas desta área abordam questões sociais econômicas, políticas e culturais, que apresentam um maior impacto local, regional e nacional, não despertando interesse de periódicos internacionais (Manoel \& Carvalho, 2011).

Desde o final da década de 1990 a avaliação da pós-graduação passou a ter como referência a internacionalização. Passou-se a quantificar o número de artigos em periódicos com alto fator de impacto publicados por docentes e discentes dos programas, tornando os artigos itens mais valorizados na avaliação (Manoel \& Carvalho, 2011). Ainda segundo os autores a produção publicada em periódicos brasileiros é desvalorizada independentemente de seu impacto científico e de sua importância social, considerando as necessidades nacionais, regionais e locais. E apesar de todo esforço para se estabelecer novos parâmetros de avaliação, os livros são também desvalorizados junto aos programas de pós-graduação. 
Assim, os artigos tornam-se commodities. O importante é exportá-los para que tenham relevância internacional. Nem os próprios discentes leem os artigos e nem os próprios docentes discutem com profundidade o impacto daquele conhecimento produzido. Na busca por legitimização, a pesquisa de caráter científico tem sido o ponto de convergência do trabalho acadêmico em Educação Física, muito em função do apelo às publicações em larga escala e da busca por fomento financeiro público ou privado. Influenciada pelo paradigma cartesiano, que no modelo biomédico considera o corpo como uma máquina a intervenção científica só pode ser realizada a partir da divisão do organismo em múltiplas partes, que apesar de se relacionarem, necessitam de especialistas para cada uma delas.

Esta política de avaliação centrada em critérios originários das ciências biológicas, acaba induzindo a uma caracterização da Educação Física regida por temas que apresentam maior probabilidade de publicação, fazendo com que assim neste jogo de poder e saber (Foucault, 2006) as pesquisas nas ciências físicas e biológicas sejam mais valorizadas, e na busca obsessiva em alcançar níveis internacionais acabem desprezando a pesquisa nas subáreas sociocultural e pedagógica.

Com processo de gestão da própria saúde, a otimização dos padrões corporais acabou tornando-se um parâmetro de medida de valor para o homem dito pós-moderno e criou novas formas de relação consigo e com o outro, onde o modelo ideal de sujeito baseia-se na performance física e na adoção de comportamentos regrados em preceitos higiênicos, com o objetivo de obter melhor forma física e maior longevidade (Ortega \& Zorzaanelli, 2010).

Objetivando não somente a sujeição dos corpos, mas sobretudo o controle preciso da população através de dados estatísticos. Devido a associação de diferentes hábitos cotidianos a doenças crônico-degenerativas, com a massificação das informações sobre promoção da saúde, os indivíduos têm se apropriado dos discursos técnicos-científicos médicos e obtido subsídios para avaliação e gestão da própria saúde.

Neste contexto, entretanto, parece diminuir o espaço para as questões sociais, psicológicas e culturais do processo corposaúde-doença. É possível perceber então uma Educação Física que vem abandonado a formação pedagógica para reduzi-la a uma formação científica, com competências voltadas cada vez mais para teoria e não para prática (Gaya, 2014).

Os cursos de pós-graduação stricto sensu em Educação Física no Rio de Janeiro vêm sofrendo mudanças históricas com base nos discursos que vem sendo produzidos na sociedade sejam pelos sujeitos da área ou não, pois atualmente a Educação Física apoia-se na valorização social da ciência para alcançar sua legitimidade acadêmica e social (Bosseler \& Fraga, 2016). A verdade científica tornou-se a garantia de verdade de qualquer área de conhecimento. Os estudos filosóficos em Educação Física, por exemplo, perderam espaço para estudos fisiopatológicos, bioquímicos e biomecânicos. Tal fato pode estar relacionado a construção de um campo científico com agentes de conservação (Bourdieu, 2004) ou mesmo com dispositivos de poder (Foucault, 2014) que produzem efeitos de afastamento das subáreas sociocultural e pedagógica da base epistemológica e da prática profissional do graduado ou pós-graduado em Educação Física.

\section{Considerações Finais}

Analisando pela perspectiva genealógica foucaultiana que nos permite refletir sobre as tecnologias e dispositivos de saber-poder, tal como ocorreu com a medicina moderna, a biologização da Educação Física nos permite compreender que à medida que a Educação Física se aproxima do modelo hegemônico de ciência com suas regras e discursos, conquista maior legitimidade nos campos científico e social. A biologização confere também um caráter utilitarista a medida que faz com que os indivíduos se apropriem do discurso científico para gerirem a própria saúde. No entanto esta biologização por seu caráter reducionista afasta a Educação Física do seu universo complexo transdisciplinar, que nos permite contextualizar e fazer uma análise interpretativa da realidade, principalmente em algumas questões observadas dentro da prática pedagógica, como a violência, o sofrimento, questões de gênero, intolerância entre outros problemas provenientes das relações humanas, mas que apesar disso não lhe confere o mesmo prestígio que o adquirido com a valorização das ciências biomédicas. 
Research, Society and Development, v. 10, n. 9, e7410917801, 2021

(CC BY 4.0) | ISSN 2525-3409 | DOI: http://dx.doi.org/10.33448/rsd-v10i9.17801

\section{Referências}

Bossle, C. \& Fraga, A.(2016). A racionalidade biomédica desportiva e a materialização do fazer científico na matriz curricular do curso de Educação Física da UFRGS. Movimento, 22 (3), 877-888.

Bourdieu, P. (2004) Os usos sociais das ciências: por uma sociologia clínica do campo científico. Unesp.

Bracht, V. (1997) Sociologia crítica do esporte: Uma introdução UFES, Centro de Educação Física e Desportos.

Castellani Filho, L. (1988) Educação física no Brasil: a história que não se conta. Papirus.

Gaya, A. (2014) Educação Física: ordem, caos e utopia. Casa da Educação Fìsica.

Fórum dos Pesquisadores das subáreas Sociocultural e Pedagógica. (2015) Cenários de um descompasso da pós-graduação em Educação Física e demandas encaminhas à CAPES. http://www.cbce.org.br/noticiasdetalhe.php?id=1074.

Foucault, M. (1989) La volonté de savoir. In: Foucault, M. Résumé des cours (1970-1982). Julliard.

Foucault, M. (2006). Poder e Saber. Motta MB. (Orgs. Trad.). Michel Foucault. Estratégia, Poder-Saber. Ditos \& Escritos IV. $2^{\text {a }}$ Edição Rio de Janeiro: Forense Universitária.

Foucault, M. (2008) A Arqueologia do Poder. (7a ed.), Forense Universitária.

Foucault, M. (1999) História da Sexualidade I: a vontade de saber. (13a ed.), Graal Edições.

Foucault, M. (2014) Microfísica do Poder. (28 ed.), Paz \& Terra.

Kokubun, E. (2006). Pós-Graduação em Educação Física. Revista. Brasileira de Educação Física e Esporte, 20 (5), 31 -33.

Lüdorf, S. (2002). Panorama da pesquisa em educação física da década de 90: análise dos resumos de dissertações e teses. Revista da Educação Física/UEM, $13(2), 19-25$.

Luz, M. (2007) Educação Física e saúde coletiva: papel estratégico da área e possibilidades quanto ao ensino na graduação e integração na rede de serviços públicos de saúde. In: Fraga, A. B. \& Wachs, F. Educação Física e saúde coletiva: políticas de formação e perspectivas de intervenção. Ed. da UFRGS.

Manoel, E. \& Carvalho, Y. (2011) Pós-graduação na educação física brasileira: a atração (fatal) para a biodinâmica. Educação e Pesquisa, 37 (2), $389-406$.

Ortega, F. \& Zorzanelli, R. (2010). Corpo em evidência: a ciência e a redefinição do humano. Civilização Brasileira.

Paiva, F. (2004) Contribuições da Hygiene à escolarização da Educação Física. Movimento, 10 (3), 53-75.

Telles, S., Lüdorf, S. \& Pereira, E. (2017) Subáreas Sociocultural e Pedagógica na Educação Física: Ainda a caminho do fim. Telles, S., Lüdorf, S. \& Pereira,

E. Pesquisa em educação física: perspectivas sociocultural e pedagógica em foco. Autografia.

Thomas, J., Nelson, J. \& Silverman, S. (2012). Métodos de pesquisa em atividade física. (6a ed.), Artmed.

Triani, F. \& Telles, S. (2019). A pós-graduação stricto sensu em educação física no Rio de Janeiro: desafios para a formação acadêmica e a produção científica a partir das possibilidades de publicação. Journal os Physical Education, 30, e3050x.

Triani, F., Barros, G., Magalhães Júnior, C. \& Telles, S. (2019). As representações sociais de bacharelandos sobre ser profissional de educação física. Journal os Physical Education, 30, e3032x.

Triani, F., Magalhães Júnior, C. A. \& Novikoff, C. (2017). As representações sociais de estudantes de educação física sobre a formação de professores. Movimento, 23 (2), 575-586.

Williams, J. (2012). Pós-estruturalismo (Coleção Pensamento Moderno). Vozes. 